\title{
Debonding Techniques - A Review
}

\author{
Purva Vinod Kumar Dhannawat ${ }^{1}$, Rizwan Gilani², Sunita S. Shrivastav ${ }^{3}$, Ranjit H. Kamble ${ }^{4}$, \\ Shriya Prakash Murarka ${ }^{5}$, Shruti Satish Rathi ${ }^{6}$, Rozina Vishnani ${ }^{7}$ \\ 1, 2, 3, 4, 5, 6, 7 Department of Orthodontics and Dentofacial Orthopaedics, \\ Sharad Pawar Dental College, Sawangi, Wardha, Maharashtra, India.
}

\section{ABSTRACT}

\section{BACKGROUND}

The overall success of orthodontic treatment depends on both bonding as well as debonding techniques. The debonding procedure in orthodontics consists of removal of the attachments (brackets, bondable tubes \& buttons) as well as all the adhesive resin from the teeth without causing any permanent damage and to restore the teeth to their pre-treatment stage. The demand for more aesthetic appliance led to the introduction of direct bonding techniques and has made banded attachments almost obsolete in present-day orthodontics. These procedures should not be painful to the patient or damaging to enamel and to obtain these objectives a correct debonding technique is of fundamental importance. The debonding procedure is as essential as bonding for the overall success of the orthodontic treatment. In an attempt to increase the bond strength of orthodontic appliances, we have neglected the fact that these appliances have to be debonded at the end of the treatment. In orthodontics debonding refers to debracketing that is removal of brackets, bondable tubes, buttons, and the adhesive used to bond as well as to restore the form and surface of tooth to its best possible original form by avoiding any type of iatrogenic damage. For achieving such objectives, an accurate debonding procedure is of utmost importance, else it could be needlessly lengthy and painful to the patient and damaging to the enamel. Many researches have been carried out to conclude the best techniques for debonding which will give an ideal finish for the tooth when treatment is terminated. The debonding procedure is mostly done by mechanical means, but its technique should be varied according to the bracket material and type.

\section{KEY WORDS}

Debonding Techniques, Metal Brackets, Ceramic Brackets, Enamel Damage
Corresponding Author: Dr. Purva Vinodkumar Dhannawat, Sharad Pawar Dental College, Sawangi, Wardha, Maharashtra, India. E-mail: pvdhannawat@gmail.com

DOI: $10.14260 /$ jemds/2021/694

How to Cite This Article: Dhannawat PV, Gilani R, Shrivastav SS, et al. Debonding techniques - a review. J Evolution Med Dent Sci 2021;10(38):34303435, DOI: 10.14260/jemds/2021/694

Submission 26-04-2021,

Peer Review 30-06-2021,

Acceptance 07-07-2021,

Published 20-09-2021.

Copyright (C) 2021 Rizwan Gilani et al. This is an open access article distributed under Creative Commons Attribution License [Attribution 4.0 International (CC BY 4.0)] 


\section{BACKGROUND}

The past 30 years have seen major changes in the evolution of orthodontic materials. Due to increased awareness, patients are not only demanding high-quality orthodontic treatment, but they also want the treatment to be minimally invasive and more comfortable with an aesthetically pleasing outcome. The demand for more aesthetic appliance led to the introduction of direct bonding techniques and has made banded attachments almost obsolete in present-day orthodontics. However, with extensive utilization of direct bonding materials, concerns were raised over removal of residual bonding resin from the teeth surfaces most effectively without damaging the enamel. ${ }^{1}$ The debonding procedure is as essential as bonding for the overall success of the orthodontic treatment. In an attempt to increase the bond strength of orthodontic appliances, we have neglected the fact that these appliances have to be debonded at the end of the treatment. In orthodontics debonding refers to debracketing that is removal of brackets, bondable tubes, buttons, and the adhesive used to bond as well as to restore the form and surface of tooth to its best possible original form by avoiding any type of iatrogenic damage. For achieving such objectives, an accurate debonding procedure is of utmost importance, else it could be needlessly lengthy and painful to the patient and damaging to the enamel. ${ }^{1}$ Many researches have been carried out to conclude the best techniques for debonding which will give an ideal finish for the tooth when treatment is terminated. The debonding procedure is mostly done by mechanical means, but its technique should be varied according to the bracket material and type. Mechanical debonding of the ceramic bracket is risky as it may cause bracket fracture. But for other brackets like metal, this is the most common method used. Improper methods for debonding ceramic brackets may cause injury to the dentist as well as the patient from flying fragments of bracket.2,3 No documentation has been done till date for discomfort of patient during removal of brackets; however, practical incidences indicates that "gentler" methods for removal of brackets are required. Several procedures have been described for debonding orthodontic brackets. Mouth \& teeth are the most essential aspects in facial aesthetics. Aesthetics effects which will occur due to orthodontic treatment should be controlled. ${ }^{4}$ These techniques include: Mechanical, Thermal, Ultrasonic, Chemical, and Laser debonding.

Clinical procedures -

1. Removal of bracket.

2. Removal of residual adhesive resin.

\section{Mechanical Techniques}

A. Steel Brackets

Several different procedures are there for debonding steel brackets.

\section{Debonding Plier}

This is one of the recommended techniques for debonding which is done by placing chisel shaped beaks near base of bracket \& application of peeling type of force. Due to ductility of metal brackets, the applied force is transferred to the adhesive bond, and hence breaking it. ${ }^{2}$

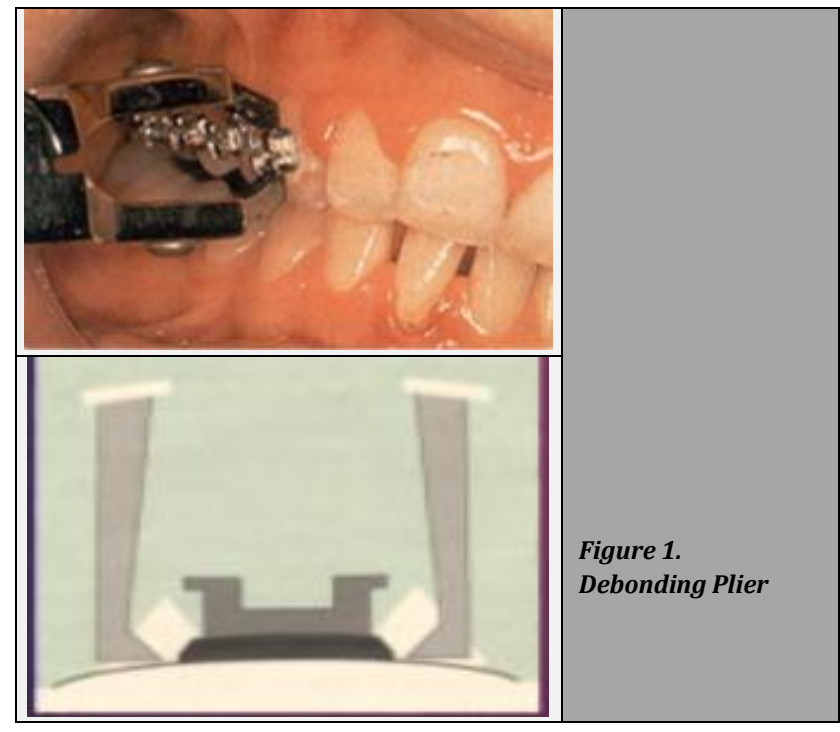

2. Lift off Debonding Instrument

There is a wire attached to the plier which is engaged under the bracket wing. Pull wire produces pulling force, both the plastic rests have to be placed on the tooth surface and light squeeze force has to be applied with handles. This pull wire is incorporated for minimal damage to the bracket so that it can be recycled if desired.5,6

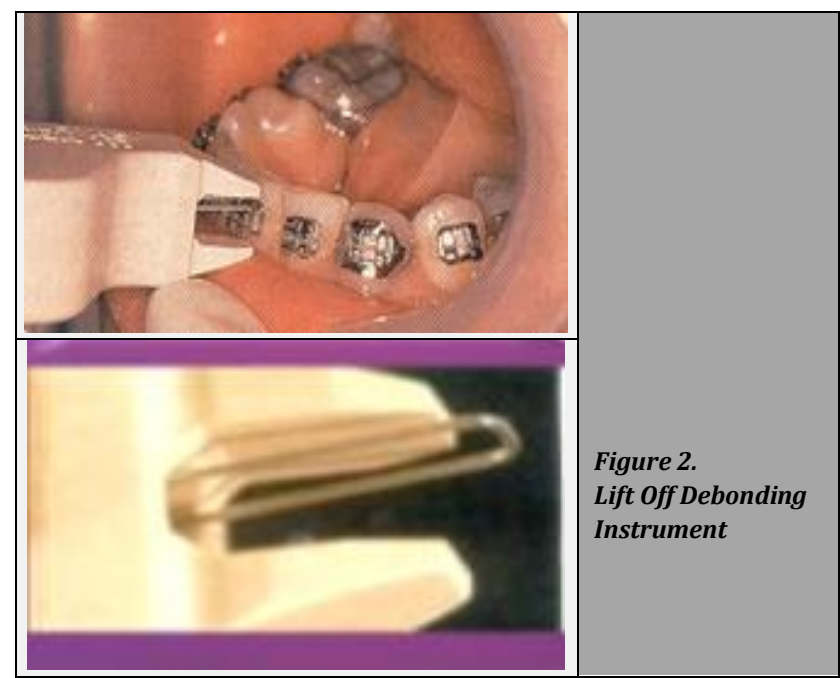

3. Ligature Cutters / Straight Cutter Plier

It is also one of the instruments to be used for debonding as per some authors.

The straight cutter plier is used for grabbing the wings of the bracket \& to apply pressure on the bracket base both mesially \& distally.

But the chance of beak damage is there which will detract from the original purpose.5,6

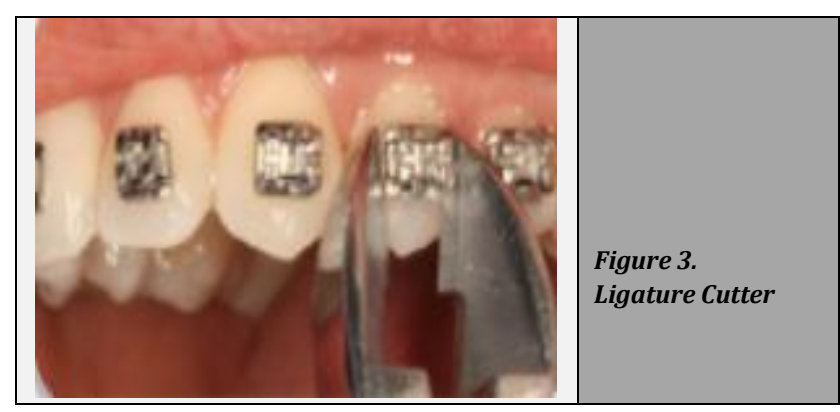




\section{Weingart Pliers}

This is another method for debonding in which wings of the bracket are squeezed together by Weingart plier. This shear force is transmitted to composite adhesive causing breakage of the bond. This method may distort the bracket wings, alteration of the slot dimension, making the bracket nonreusable. ${ }^{6}$

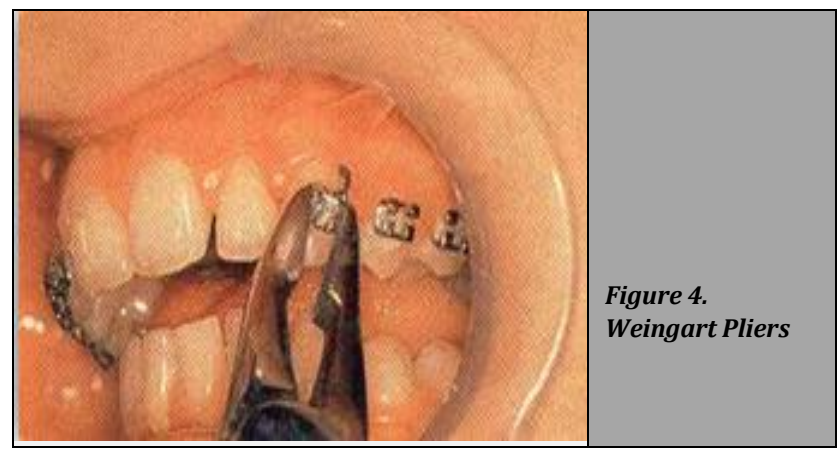

\section{B. Ceramic Brackets}

There is high risk of fracture of bracket during mechanical debonding of the ceramic bracket. Partially broken bracket has to be separated by using a diamond bur and if coolant is not used then it may be injurious to the pulp, harmful to the enamel surface as well as it is a time-consuming procedure.

Usually, horizontal enamel cracks may be seen if brackets are cut off by applying gradual pressure from tip of the twin beaked pliers near the bracket adhesive surface, $\&$ hence it is not usually recommended.7

Various manufacturers have given different recommendations on debonding for reporting the trouble of enamel fracture and cracks while deboning.

ETM 346 plier can be used to remove allure brackets as recommended by GAC (fig. 5). Bevels are given on the incisal as well as gingival edges of the allure bracket for easy insertion.

For catching any splinters from getting away while debonding procedure, an analogous plier with an additional shield is available (fig. 6).

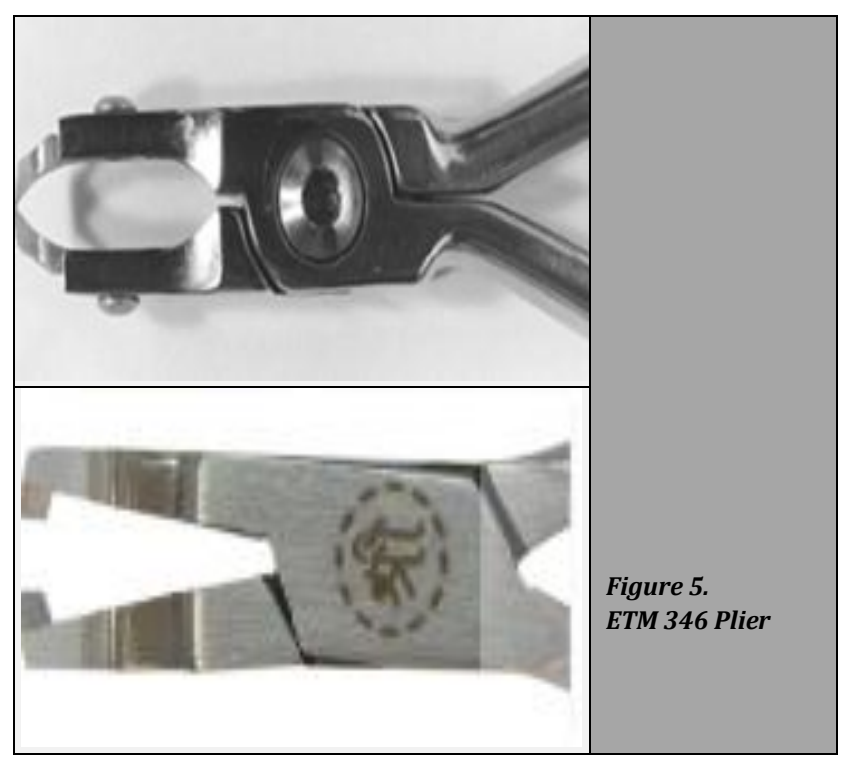

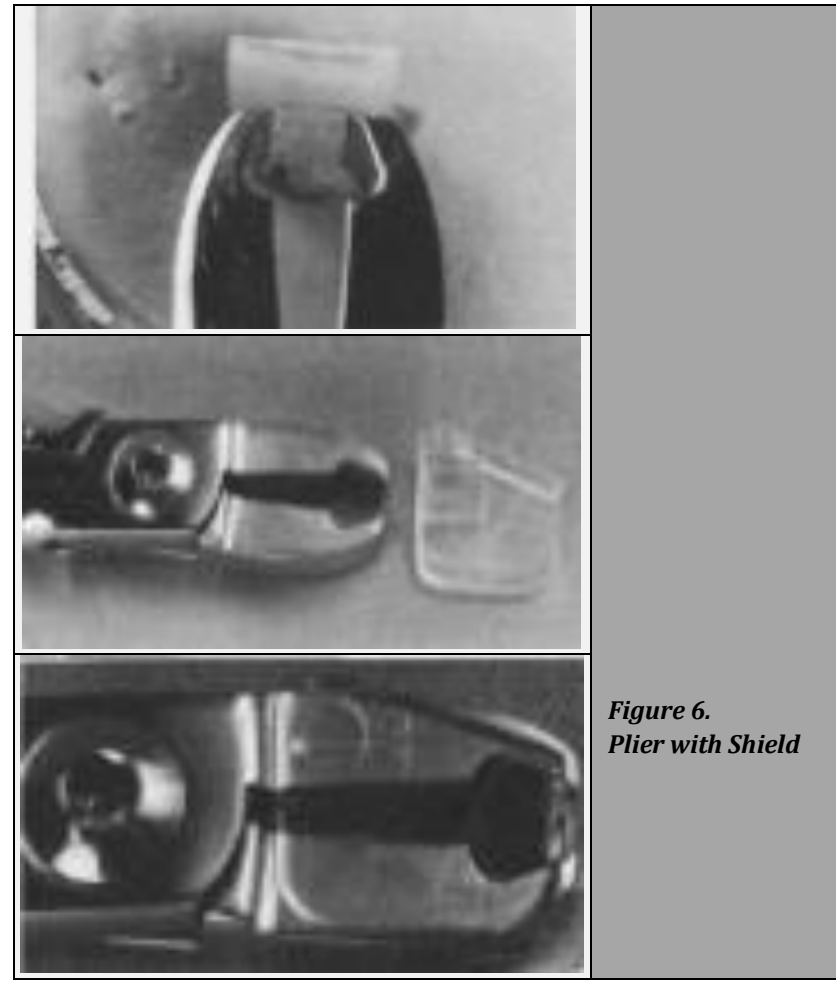

$3 \mathrm{M}$ unitek does not advise the usage of routine debonding pliers for debonding transcend 2000 bracket. They are mechanically retained over tooth surface they have strong shear strength but has a weaker tensile strength (fig. 7).

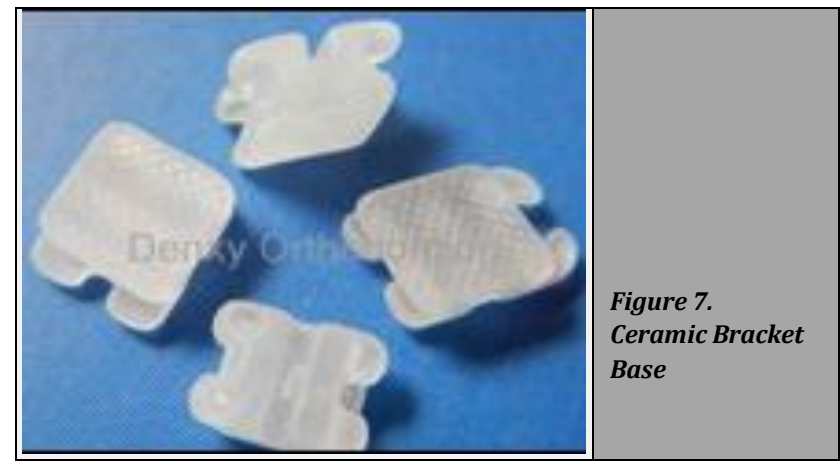

Accordingly, Unitek has introduced a new debonding tool, called the debonding wrench (fig. 8) which applies a tensile force on the bracket while debonding. The use of this debonding wrench has been shown to produce less enamel damage. ${ }^{5}$

\section{Electrothermal Debonding}

Other than the conventional method, there is an alternate technique to debond metallic brackets. ${ }^{8}$ This technique was given by Sheridan et al. and is known as electro-thermal debonding. ${ }^{9}$ It is a cordless battery device that generates heat and remove brackets from enamel surfaces. ${ }^{10}$ 

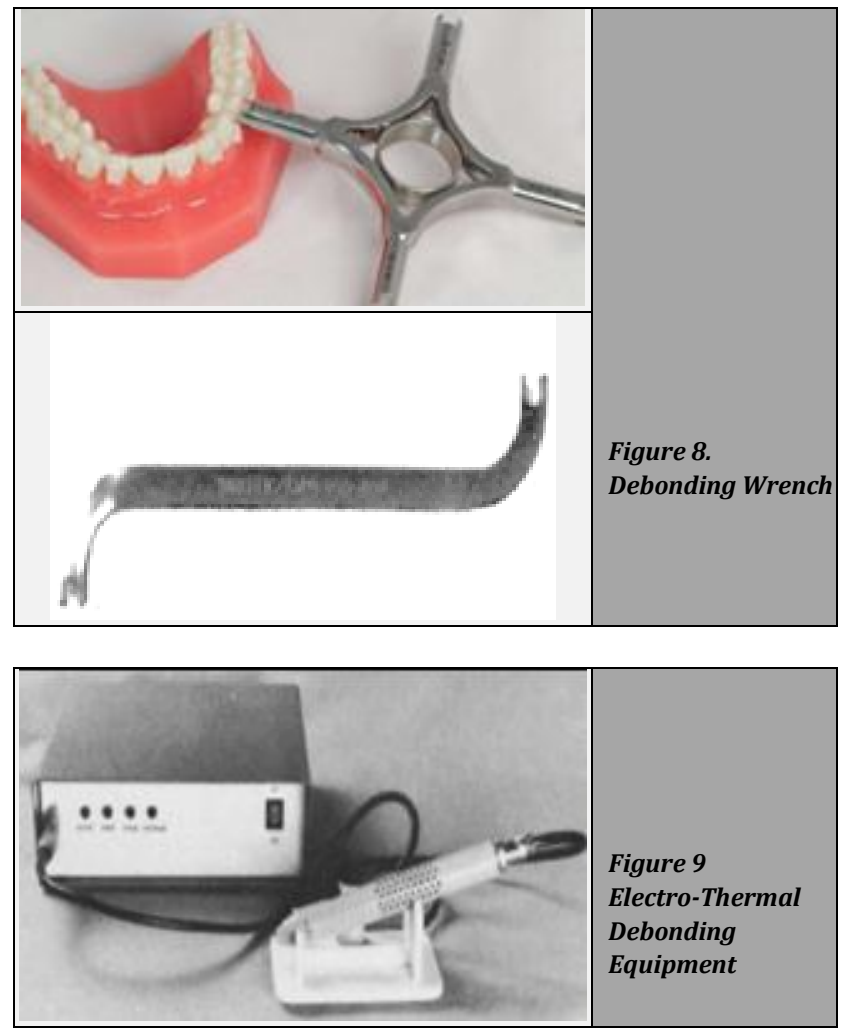

\section{Chemical Debonding}

Previously, peppermint oil has been material of choice for debonding brackets. Some authors have studied its effect ex vivo on debonding of ceramic bracket as compared to softening agents like ethanol $\&$ acetone. ${ }^{11}$

Lowest mean \& maximum debond force (77 \& $114 \mathrm{~N}$ resp.) was observed with low retained resin when placed for 1 hour in peppermint oil. Bracket fractures are still there but no confirmation of enamel fracture was seen with any of the group. ${ }^{12}$

\section{Ultrasonic Debonding}

One more alternate technique is there which uses ultrasonic tips for debonding of brackets (fig 10). The KJS tip, (KJC and KJS Dentsply Int. York, Pa.) is initially placed at an incisal portion of the bracket for removal. The bevel of straight chisel is positioned towards the bracket itself. 13

After tip placement, ultrasonic unit is made active while moving the tip mesially as well as distally. The motion will immediately create a groove in the composite. ${ }^{14}$ Once 0.5 $1 \mathrm{~mm}$ purchase point was gained, a rocking motion was then included till the bond failure occurred. Use of KJS with KJC tip was used alternatively for bracket removal. ${ }^{13}$

On gaining approximately a 0.5 to $1 \mathrm{~mm}$ "purchase point," a rocking motion was then incorporated until bond failure occurred. Alternating the use of the KJS with the KJC tip facilitated bracket removal. It was seen that though the chances of bond failure were high at enamel adhesive interface while using ultrasonic technique, there were less chances of damage to enamel. ${ }^{13}$

In the conventional technique, the force generated for bond failure is much more when compared to levels of force generated with ultrasonic tips but the drawback is that time required for the ultrasonic method is much more.

\section{Laser Debonding}

Optic laser technology was discovered in 1960s with the invention of ruby lasers. USFDA in 1980s \& early 1990s approved the introduction of laser in dentistry. Lasers use began in early 1990s for debonding of ceramic brackets.
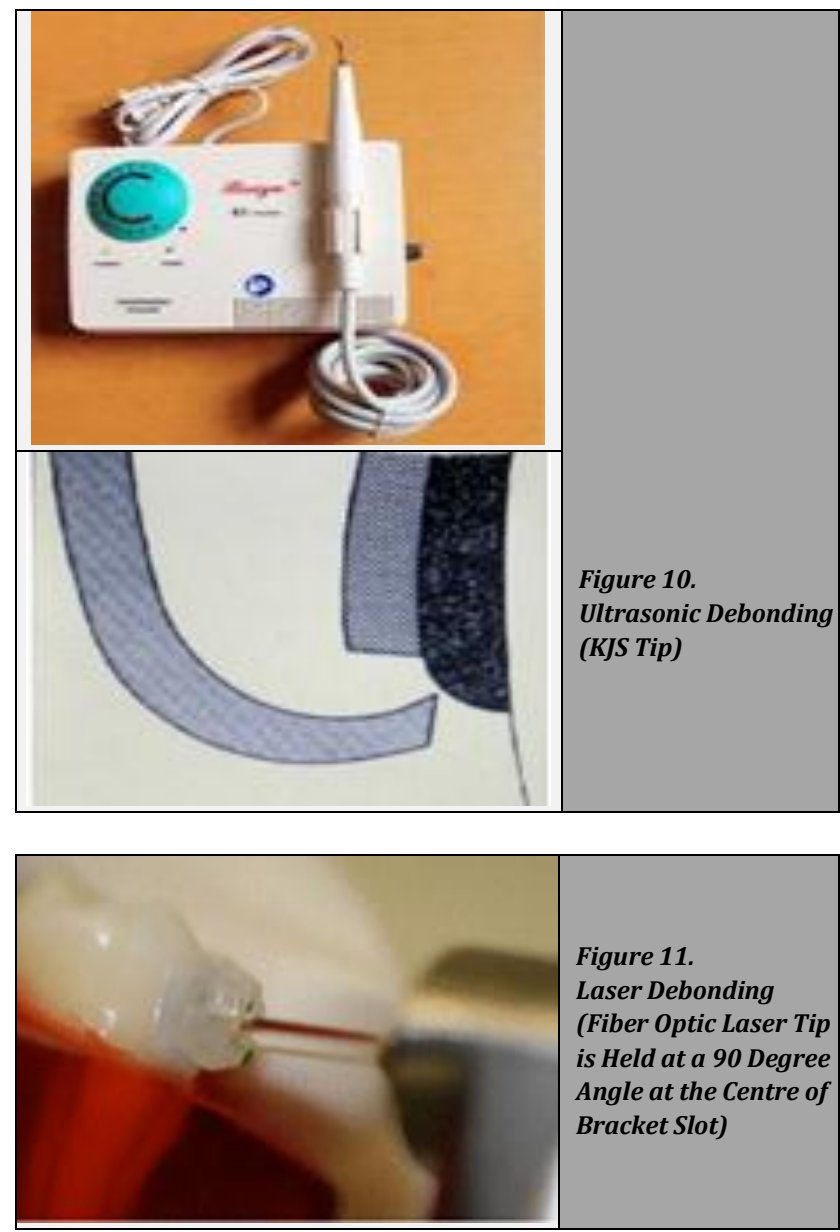

Mechanism of laser debonding (fig. 11). Adhesive resin can be degraded by laser energy by 3 methods: ${ }^{15}$

- Thermal softening

- Thermal ablation

- Photo ablation.

The laser generates heat because of which bonding agent gets soften called thermal softening. Clinically, this results in the succumbing of bracket to gravity \& slipping off from the surface of tooth. This is time-consuming as well as a slow process. So, the heat generated during this may result in temperature rise in both tooth and bracket. ${ }^{15}$

When the heating is quick enough to heave the temperature of resin to its vaporization temperature, thermal ablation takes place that too prior to debonding by thermal softening. As a result, debonding of bracket from tooth surface occurs. ${ }^{16}$ Photo ablation also results in debonding of bracket from the tooth surface. Interaction of high-level laser light with the adhesive resin causes an increase in the energy level of a bond between the atoms of adhesive to quickly rise above their dissociation energy level leading to decomposition of the material. ${ }^{17}$ It is suggested that the debonding of monocrystalline brackets occurs by ablation, though for polycrystalline brackets, thermal softening has been the reason for debonding. It is shown that by using lasers, debonding force is extensively reduced while using a Bis GMA adhesive resin. ${ }^{18}$ 


\section{Finishing Procedures, After Debonding}

For an orthodontist, to remove directly bonded attachments \& to finish off underlying enamel, some difficulty is faced by practitioner. ${ }^{19}$ And a proper finishing procedure is a must for the overall success of the treatment. It has been proved that the use of debonding pliers, scalers \& diamond finishing burs for removing the residual resin after debonding procedure should not be used as it may lead to deep gouges in enamel.20 It is recommended to use a tungsten carbide bur 12-bladed (fig. 12) at higher speed along with ample air cooling to take out the bulk resin and the underneath enamel \& residual resin is finished by graded polishing discs and ceramic wheels by way of light pressure \& ample air cooling. ${ }^{21}$

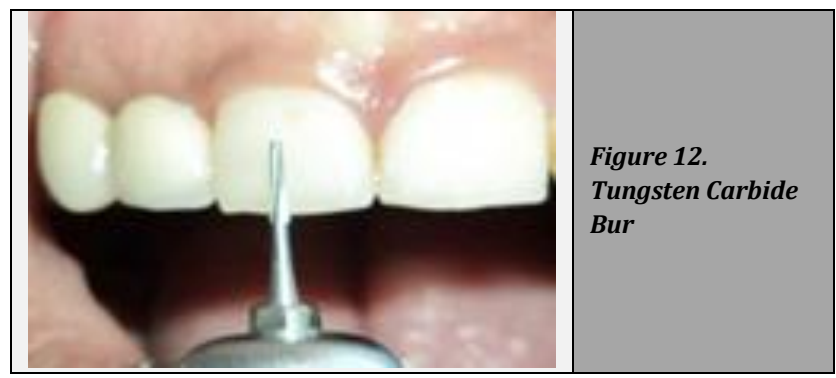

The ultimate finishing should be carried out by water slurry of pumice applied with a rubber cup. ${ }^{21}$

Recommended technique for debonding -

- $\quad$ Step 1 - Remove ligature wires as well as elastic ties to free archwire and remove arch wire with plier.

- Step 2 - Brackets are gripped at mesial and distal tie wing, one by one with debonding plier, stabilize the tooth from the lingual side by using light finger pressure.

- $\quad$ Step 3 - Stabilizing your elbow and arm, gently squeeze bracket and then lift plier outwardly at a 45-degree angle. This stroke causes bottom of the bracket to strip away from the bonding material, lifting the edges of the bracket base.

- Step 4 - Pliers should be held such that its indentation should engage in the wings of bracket for secured grip.

- $\quad$ Step 5 - Chances of slot distortion is there due to lateral force application from pliers. To avoid this, debonding can be done by keeping wire as it is in slot.

- $\quad$ Step 6 - Shear forces applied are transmitted to composite adhesive causing breakage of bond. Bond breakage takes place at bracket and adhesive interface. Forces should be applied at interface rather than bracket itself.

- Step 7 - The remaining adhesive after debonding procedure should be removed by using different instruments. a) A 12-fluted tungsten carbide bur (Dentaurum no. 123-604, Springe, Germany) used at lower speed by air cooling or a tungsten carbide bur or an ultrafine diamond finishing bur. b) Egg- or footballshaped finishing burs work well for lingual concavities, but must be used with care on convex surfaces.

- $\quad$ Step 8 - Check all the surfaces of teeth for any left-out resin adhesive with dental explorer. If there, then clean the enamel surface until it is smoothened.

- $\quad$ Step 9 - Water slurry of pumice should be used along with a rubber cup for the final finishing.

\section{DISCUSSION}

Patients today are very much conscious about their appearance, especially smile. Therefore, there has been an increased demand for alignment of the malposed teeth using orthodontic treatment, even in older adults. ${ }^{22}$ As stated above, a correct debonding technique is of utmost importance for the success of orthodontic treatment. Various authors have recommended different techniques for debonding brackets but none of the techniques offer totally satisfactory results. Each technique has its advantage and disadvantage.

Mechanical methods are most commonly used as they can be easily done chair-side and are less time consuming, but they involve a greater risk of enamel fracture. It is more difficult to debond ceramic brackets as compared to metal brackets because aluminum oxide ceramic brackets contain inert contents which makes chemical solidity among the ceramic base \& the adhesive resin unworkable.

Thus, a chemical mediator (silane coupling agent) is used amid the adhesive resin \& the bracket base. Silica glass is layered at the base of every bracket in order to promote the bond with silanol function group of the silane molecule. Bracket based adhesive interface, being one of the most common location where bond failure occurs during debonding procedure in past, has been replaced by enamel adhesive interface (less desirable site) due to increase in adhesion among resin \& ceramic bracket base.

Also, while loading, stainless steel tends to lengthen approximately $20 \%$ of that of its original length before failing, whereas sapphire tends to lengthen less than $1 \%$ before failing. Therefore, ceramics have higher tendency to fracture when compared to metals under similar conditions during the debonding procedure.

Clinically, more visible near the tie wing area. Electrothermal debonding offers a good alternative to mechanical debonding but involves the high cost of equipment and a greater risk of thermal damage to the pulp. Though ultrasonic debonding can be done easily chair-side, it is very time-consuming, and can damage the scalar tips and enamel surface. Laser debonding is said to have no damaging effect on pulp, but it involves a high cost of equipment and also the potential danger of laser irradiation to the patient \& the operator. The poly and mono-crystalline brackets debond differently during laser debonding.

The different nature observed is because of alteration in design of the 2 brackets and also alteration in their dissimilar microscopic structure.

Though various techniques have been described for debonding, a mechanical method still remains the preferred method for both metal \& ceramic brackets. It should be done with proper debonding instruments as prescribed by the manufacturers. Proper finishing procedure should also be carried out after debonding in order to achieve a satisfactory finish at the closing stage of the treatment \& restoration of the tooth surface to its pre-treatment condition.

The finishing procedure should include removal of leftover adhesive and restoration of the tooth structure to its pretreatment condition along with proper fluoride therapy, teeth whitening, remineralization, and restorative procedures for correcting the enamel tear-outs and white spot lesions that are commonly seen at the end of the treatment. 


\section{CONCLUSIONS}

The concept of non-maleficence is embodied by the phrase, "first, not harm". Debonding procedure if not done properly with correct technique, can cause iatrogenic harm to the patient involving enamel loss and its complications. Further, the orthodontist also cannot reuse the distorted/fractured brackets caused by incorrect techniques. Hence, a proper debonding technique with proper finishing procedure can be a cherry on the cake for a good finished orthodontic case.

Financial or other competing interests: None.

Disclosure forms provided by the authors are available with the full text of this article at jemds.com.

\section{REFERENCES}

[1] Zachrisson BU, Büyükyilmaz T. Bonding in Orthodontics. In: Graber L, Vanarsdall R, Vig K, et al. eds. Orthodontics: Current principles and techniques. $5^{\text {th }}$ edn. Mosby 2011: p. 749-56.

[2] Oliver RG, Pal AD. Distortion of edgewise orthodontic brackets associated with different methods of debonding. Am J Orthod Dentofacial Orthop 1989;96(1):65-71.

[3] Coley-Smith A, Rock WP. Distortion of metallic orthodontic brackets after clinical use and debond by two methods. Br J Orthod 1999;26(2):135-9.

[4] Pisulkar SK, Agrawal R, Belkhode V, et al. Perception of buccal corridor space on smile aesthetics among specialty dentist and layperson. Journal of International Society of Preventive \& Community Dentistry 2019;9(5):499-504.

[5] Bishara SE, Trulove TS. Comparisons of different debonding techniques for ceramic brackets: an in vitro study. Part I. Background and methods. Am J Orthod Dentofacial Orthop 1990;98(2):145-53.

[6] Bishara SE, Olsen ME, Von Wald L. Evaluation of debonding characteristic of a new collapsible ceramic bracket. Am J Orthod Dentofacial Orthop 1997;112(5):552-9.

[7] Diaz C, Swartz M. Debonding a new ceramic bracket: a clinical study. J Clin Orthod 2004;38(8):442-5.

[8] Sheridan JJ, Brawley G, Hastings J. Electrothermal debracketing Part II. An in vivo study. Am J Orthod Dentofacial Orthop 1986;89(2):141-5.

[9] Takla PM, Shivapuja PK. Pulpal response in electro thermal debonding. Am J Orthod Dentofacial Orthop 1995;108(6):623-9.
[10] Kearns HP, Sandham JA, Jones BW, et al. Electrothermal debonding of ceramic brackets: an ex vivo study. $\mathrm{Br}$ J Orthod 1997;24(3):237-42.

[11] Jost-Brinkmann PG, Radlanski RJ, Artun J, et al. Risk of pulp damage due to temperature increase during thermo debonding of ceramic brackets. Eur J Orthod 1997;19(6):623-8.

[12] Larmour CJ, McCabe JF, Gordan PH. An ex-vivo investigation into effect of chemical solvents on debonds behavior of Ceramic orthodontic brackets. Br J Orthod 1998;25(1):35-9.

[13] Krell KV, Courey JM, Bishara SE. Orthodontic bracket removal using conventional and ultrasonic debonding techniques, enamel loss and time requirements. Am J Orthod Dentofacial Orthop 1993;103(3):258-66.

[14] Luthra S, Vighnesh K, Rao N, et al. Ceramic brackets and their electrothermal debonding - an in vivo evaluation. TIBAO 1998;12(2):47-9.

[15] Tocchio RM, Williams PT, Mayer FJ, et al. Laser debonding of ceramic orthodontic brackets. Am J Orthod Dentofacial Orthop 1993;103(2):155-62.

[16] Azzeh E, Feldon PJ. Laser debonding of ceramic brackets: a comprehensive review. Am J Orthod Dentofacial Orthop 2003;123(1):79-83.

[17] Obata A, Tsumura T, Niwa K, et al. Super pulse CO2 laser for bracket bonding and debonding. Eur J Orthod 1999;21(2):193-8.

[18] Ma T, Marangoni RD, Flint W. In vitro comparison of debonding force and intrapulpal temperature changes during ceramic orthodontic bracket removal using a carbon dioxide laser. Am J Orthod Dentofacial Orthop 1997;111(2):203-10.

[19] Strobl K, Bahns T, Wilham L, et al. Laser aided debonding of orthodontic ceramic brackets. Am J Orthod Dentofacial Orthop 1992;101(2):152-8.

[20] Hosein I, Sherriff M, Ireland AJ. Enamel loss during bonding, debonding and cleanup with use of a self-etching primer. Am J Orthod Dentofacial Orthop 2004;126(6):717-24.

[21] Retief DH, Denys FR. Finishing of enamel surface after debonding orthodontic attachments. Angle Orthod 1979;49(1):1-10.

[22] Pakhare VV, Khandait CH, Shrivastav SS, et al. Piezosurgery ${ }^{\circledR}$-assisted periodontally accelerated osteogenic orthodontics. Journal of Indian Society of Periodontology 2017;21(5):422-6. 